\title{
Length of the Perpendicular from a given Point to a given Straight Line.
}

To obtain the length of the perpendicular from the point $P\left(x_{1}, y_{1}\right)$ to the line $a x+b y+c=0$ the following simple method, which requires only a knowledge of the formula for the area of a triangle in terms of the coordinates of the vertices, may be employed.

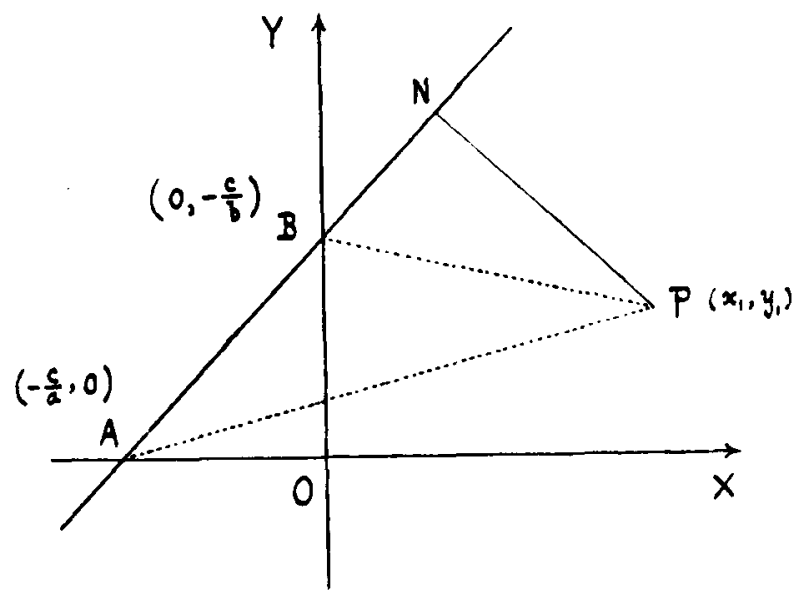

(i) The given straight line cuts the $x$-axis in the point $A\left(-\frac{c}{a}, 0\right)$, and the $y$-axis in the point $B\left(0,-\frac{c}{b}\right)$. The area, $\triangle$, of triangle $A B P$ is therefore given in sign and magnitude by

$$
\begin{aligned}
2 \Delta & =\left|\begin{array}{rr}
-\frac{c}{a}, & 0,1 \\
0, & -\frac{c}{b}, 1 \\
x_{1}, & y_{1}, 1
\end{array}\right| \\
& =\frac{c}{a b}\left(a x_{1}+b y_{1}+c\right) .
\end{aligned}
$$

Also $A B^{2}=\frac{c^{2}}{a^{2}}+\frac{c^{2}}{b^{2}}=\frac{c^{2}}{a^{2} b^{2}}\left(a^{2}+b^{2}\right)$, and if $P N$ is the perpen- 
dicular from $P$ to the line

$$
\begin{aligned}
2 \triangle & =A B \cdot P N \\
& = \pm \frac{c}{a b} \sqrt{a^{2}+b^{2}} \cdot P N .
\end{aligned}
$$

Equating these two expressions for $2 \Delta$ we have at once

$$
P N= \pm \frac{a x_{1}+b y_{1}+c}{\sqrt{\left(a^{2}+b^{2}\right)}}
$$

(ii) The special case in which the straight line passes through the origin (when $A$ and $B$ coincide with $O$, and $\Delta=0$ ) may obviously be regarded as the limiting case of (i) for $c \rightarrow 0$.

Or, if it is desired to avoid the limit-conception, we have only to note that the perpendicular from $P$ to $a x+b y=0$ is equal to the perpendicular form $O$ to the parallel through $P$, namely

$$
a\left(x-x_{1}\right)+b\left(y-y_{1}\right)=0,
$$

and that, by (i), the length of that perpendicular is

$$
\pm \frac{a\left(0-x_{1}\right)+b\left(0-y_{1}\right)}{\sqrt{ }\left(a^{2}+b^{2}\right)}= \pm \frac{a x_{1}+b y_{1}}{\sqrt{ }\left(a^{2}+b^{2}\right)} .
$$

(iii) The reader may be interested to refer to a method of finding the length of the perpendicular by projections, given by Prof. R. J. T. Bell in Mathematical Notes, No. 18 (May 1915), pp. 206-207.

\section{J. M'WHAN.}

\section{Formulae for the Construction of Right-Angled Triangles.}

Use the formulae

$$
\text { or } \quad \begin{aligned}
& {[a(a+2 b)]^{2}+\left[2 b(a+b)^{2}\right]^{2}=\left[2 b(a+b)+a^{2}\right]^{2}} \\
& {[2 a(a+b)]^{2}+[b(2 a+b)]^{2}=\left[b(2 a+b)+2 a^{2}\right]^{2} \text {. }}
\end{aligned}
$$

In these $a$ and $b$ need not be integers, and may be positive or negative.

The formulae develop into two systems of sets of triangles. 